\section{Die Abrechnung von Kontrastmitteln unter dem Eindruck des Antikorruptionsgesetzes}

\section{Einführung}

Die Abrechnung von Kontrastmitteln in der vertragsärztlichen Versorgung ist bereits in der Vergangenheit mit nicht unerheblichen strafrechtlichen Risiken für niedergelassene Radiologen verbunden gewesen. Der BGH hatte sich bereits mit Urteil vom 15.10.1991 (Az.: 4 StR 420/91) mit der Abrechnung von Radionuklidkosten durch Nuklearmediziner beschäftigt und das OLG Hamm hatte in einer Entscheidung vom 22.12.2004 (Az.: 3 Ss 431/04) die Auffassung vertreten, dass die Übernahme von Entsorgungskosten für Kontrastmittel durch Arzneimittelhersteller oder Großlieferanten in Vertragsarztpraxen den objektiven Tatbestand der Untreue und des Betruges erfüllt. Die strafrechtliche Beurteilung beruhte jedoch auf dem damaligen Abrechnungssystem der zuständigen KV für diese Sachkosten.

Seit dem Inkrafttreten des Gesetzes zur Bekämpfung von Korruption im Gesundheitswesen (BGBI.I, S. 1254) am 4.6.2016 drohen nun neue strafrechtliche Risiken für Radiologen, die jedoch anders gelagert sind, als diejenigen aufgrund von Betrug oder Untreue und hiervon zu trennen sind. Zu beachten ist, dass ein Abrechnungsverhalten, welches früher als zulässig bewertet wurde, zukünftig nach den neu eingefügten § 299a und b StGB (Bestechlichkeit und Bestechung im Gesundheitswesen) strafbar sein kann, da der rechtliche Ansatz für die Strafbarkeitsbeurteilung anders ist als bei § 263 StGB (Betrug) und § 266 StGB (Untreue).

\section{Aktueller Vorgang}

Bereits im vergangenen Jahr haben niedergelassene Radiologen unangenehme Erfahrungen im Rahmen der KM-Abrechnungen mit den neuen Strafvorschriften der Bestechlichkeit und Bestechung im Gesundheitswesen machen müssen. Unter Berufung auf diese Straftatbestände haben verschiedene Kontrastmittelhersteller und -händler in den KV-Bereichen, in denen die Abrechnung auf der Grundlage von Kontrastmittelpauschalen mit den Kassenärztlichen Vereinigungen (KVen) erfolgt, mitgeteilt, zukünftig Kontrastmittel ausschließlich zu einem Preis zu verkaufen, welcher dem pauschalen Erstattungsniveau entspricht. Eine Preisgestaltung, bei welcher die Vertragsärzte die Kontrastmittel für einen Preis unterhalb der in der Ergänzungsvereinbarung festgelegten Pauschale einkaufen und infolge der Erstattung des höheren Pauschalbetrages einen Gewinn erzielen, begründet nach ihrer Ansicht sowohl eine Strafbarkeit des Herstellers als auch des betreffenden Arztes. Die Gewinnmarge, die bei einem derartigen Modell auf Seiten der Radiologen entstehen würde, sei als „Unrechtsvereinbarung über einen aus der ärztlichen Berufsausübung resultierenden Vorteil“" im Sinne der $\S \S 299 a$ und b StGB zu werten.

Der nachfolgende Beitrag setzt sich mit der Rechtsauffassung der Kontrastmittelhersteller und -händler auseinander. Aus rechtlicher Sicht sprechen die überwiegenden Argumente dafür, dass die seitens der Vertragsärzte generierten Vorteile im Hinblick auf §299a StGB rechtlich unbedenklich sind und demzufolge weder an die KVen, noch an die Krankenkassen weitergegeben werden müssen.

\section{Kontrastmittelabrechnung innerhalb der vertragsärzt- lichen Versorgung}

Bei der Abrechnung von Kontrastmitteln in der vertragsärztlichen Versorgung ist zu differenzieren: Sie erfolgt mehrheitlich über die Sprechstundenbedarfsvereinbarung des jeweiligen KV-Bezirkes (Schleswig-Holstein, Mecklenburg-Vorpommern, Brandenburg, Berlin, Sachsen-Anhalt, Thüringen, Sachsen, Hessen, Nordrhein, Rheinland-Pfalz, Saarland und Baden-Württemberg). Demgegenüber sind die Röntgenkontrastmittel in den übrigen KV-Bezirken (Hamburg, Niedersachsen, Bremen, Westfalen-Lippe und Bayern) als Pauschale

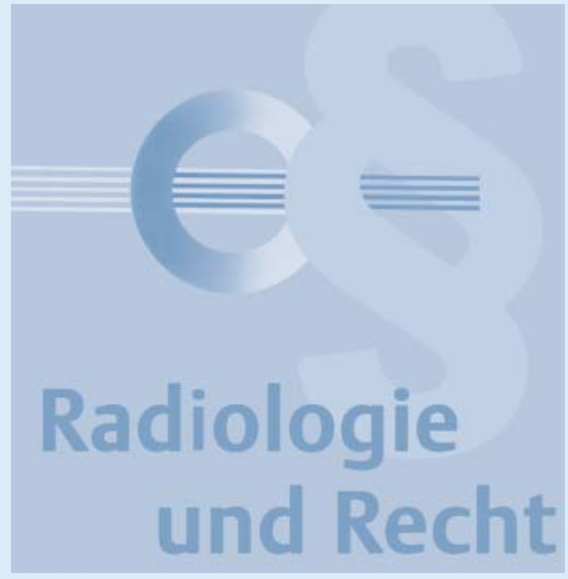

abzurechnen. Die Krankenkassen erstatten pauschal die Kosten für Kontrastmittel für den verordneten, vom Arzt tatsächlich benötigten Sprechstundenbedarf nach Maßgabe des Wirtschaftlichkeitsgebots zu mit den KV verhandelten Beträgen.

In diesem Kontext stellte das Landessozialgericht NRW (Beschl. v. 28.12.2010, Az.: L 11 KA 60/10 B ER) fest, dass es rechtlich nicht zu beanstanden sei, den Verbrauch von Kontrastmitteln einer pauschalierenden Regelung zu unterwerfen. Dies bedeutet im Umkehrschluss, dass der Vertragsarzt, der das Kontrastmittel unterhalb der Pauschalbeträge einkaufen kann oder vom Hersteller sonstige Vorteile im Rahmen des Einkaufes erhält, nicht verpflichtet ist, den günstigen Einkaufspreis oder die erlangten Vorteile an die Krankenkassen weiter zu geben, da die Krankenkassen zur Zahlung des Pauschalbetrages verpflichtet sind. Ebenso verstößt die Einführung einer Pauschalregelung im Bereich der Kostenerstattung für Kontrastmittel nach Auffassung des erkennenden Senates nicht gegen das ärztliche Berufsrecht:

„(3) Auch einen Verstoß gegen das ärztliche Berufsrecht vermag der Senat entgegen der Auffassung der Antragstellerin nicht zu erkennen. Sie meint, die Anreizstruktur der Kontrastmittelpauschalen gehe dahin, dass der Arzt das jeweilige Mittel nicht nach Indikation, sondern nach dem potentiellen Gewinnanteil auswählt. Dem ist schon deswegen nicht zu folgen, weil jedes Handeln des Vertragsarztes im Bereich der GKV [dem Wirtschaftlichkeitsgebot nach § 12 Abs. 1 SGB V] unterworfen ist und der Senat derzeit keinen Anlass für die 
Annahme sieht, dass Vertragsärzte sich dem gleichsam flächendeckend entziehen. Zudem ist einer pauschalierenden Kostenerstattung naturgemäß eigen, dass die realen Kosten höher, aber auch niedriger liegen können. Aus diesen Zusammenhängen herzuleiten, Vertragsärzte würden Kontrastmittel nunmehr nicht indikationsbezogen sondern nur noch nach pekuniären Gesichtspunkten beziehen, erachtet der Senat als überzogen. Ungeachtet dessen führt die Pauschalierung dazu, dass Vertragsärzte - allerdings wiederum unter Beachtung der Vorgaben des § 12 Abs. 1 SGB $V$ - tendenziell dazu neigen werden, das preislich günstigere Mittel zu beziehen. Das wiederum wäre ein legitimes Ziel der Ergänzungsvereinbarung."

Die Preisvorteile, die ein Vertragsarzt aufgrund eines unterhalb der Pauschale liegenden Einkaufspreises erhält, stehen daher im Ergebnis dem Vertragsarzt zu. Ein Anspruch auf Weitergabe der Preisvorteile besteht seitens der Krankenkassen aufgrund der zwischen der KV und den Krankenkassen vereinbarten Pauschalvergütungen für Kontrastmittel nicht.

\section{Strafbarkeit nach §299a StGB (Bestechlichkeit im Gesundheitswesen)}

\author{
Gemäß § 299a StGB wird bestraft,
}

„[w]er als Angehöriger eines Heilberufs [- mithin als Arzt -] im Zusammenhang mit der Ausübung seines Berufs einen Vorteil für sich oder einen Dritten als Gegenleistung dafür fordert, sich versprechen lässt oder annimmt, dass er

1. bei der Verordnung von Arznei-, Heiloder Hilfsmitteln oder von Medizinprodukten,

2. bei dem Bezug von Arznei- oder Hilfsmitteln oder von Medizinprodukten, die jeweils zur unmittelbaren Anwendung durch den Heilberufsangehörigen oder einen seiner Berufshelfer bestimmt sind, oder

3. bei der Zuführung von Patienten oder Untersuchungsmaterial

einen anderen im inländischen oder ausländischen Wettbewerb in unlauterer Weise bevorzuge“.
Spiegelbildlich zu § 299a StGB sieht § 299b StGB die Strafbarkeit der Bestechung im Gesundheitswesen vor. In besonders schweren Fällen der §299a und §299b StGB droht $\S 300$ StGB eine Freiheitsstrafe von drei Monaten bis zu fünf Jahren an. Ein besonders schwerer Fall liegt in der Regel vor, wenn sich die Tat auf einen Vorteil großen Ausmaßes bezieht oder der Täter gewerbsmäßig handelt oder als Mitglied einer Bande, die sich zur fortgesetzten Begehung solcher Taten verbunden hat. Speziell die Qualifikation des gewerbsmäßigen Handelns wird in der Praxis oftmals erfüllt sein.

Mit den Straftatbeständen wird ein doppelter Rechtsgüterschutz bezweckt. Neben dem „Schutz des fairen Wettbewerbs“ soll das „Vertrauen des Patienten in die Integrität heilberuflicher Entscheidungen“ geschützt werden. Mittelbar soll §299a StGB zudem dem Schutz der Vermögensinteressen der Wettbewerber im Gesundheitswesen, der Patienten und der GKV dienen.

\section{Bestehen eines Vorteils}

Da es sich bei §299a StGB um ein abstraktes Gefährdungsdelikt handelt, muss der Vorteil nicht tatsächlich eingetreten sein. Demensprechend sind nicht nur die Annahme, sondern bereits das Fordern sowie das Sich-Versprechen-Lassen eines „Vorteils“ durch den Arzt strafbewährt.

Unter einem „Vorteil“ ist jede Zuwendung zu verstehen, auf die der Täter (mithin der Arzt) keinen Rechtsanspruch hat und die seine wirtschaftliche, rechtliche und persönliche Lage objektiv verbessert (vgl. etwa BGH, Urt.v. 11.4.2001, Az.: 3 StR 503/00). Insoweit deckt sich der Vorteilsbegriff mit dem Vorteilsbegriff zu §31 Abs. 1 Musterberufsordnung-Ärzte. Bei strenger Betrachtung lässt sich vertreten, dass eine Strafbarkeit bereits mangels eines nachweisbaren Vorteils ausscheidet. Günstige Einkaufskonditionen stellen für sich genommen keinen Vorteil dar, da sie zu keiner Verbesserung der Lage des Empfängers beitragen. Vielmehr ist die Zahlung eines Einkaufspreises etwas nachteiliges, da bestehendes Vermögen vermindert wird. Durch den Erwerb der Kontrastmittel wird diese Vermögensminderung wieder ausgeglichen. Ein Vorteil verbleibt aber nur dann, wenn die Kontrastmittel einen höheren Wert hätten, als dafür gezahlt werden musste. Davon ist im Allgemeinen nicht auszugehen, weil kein Händler seine Ware unter deren Wert verkaufen wird, da er anderenfalls mit dem Verkauf keinen Gewinn mehr erzielen würde.

Zugleich entsteht ein Vorteil im strafrechtlichen Sinne auch dann nicht, wenn der Vertragsarzt später die Kontrastmittelpauschale abrechnet und den Differenzbetrag zwischen dem Einkaufspreis und der pauschalen Erstattungssumme einbehält. Denn nach der Definition eines Vorteils darf auf die Leistung kein Anspruch bestehen. Ein solcher Anspruch auf die Erstattung der Kontrastmittelpauschale ergibt sich aber aus der Ergänzungsvereinbarung zur Sprechstundenbedarfsvereinbarung (SSB-Vereinbarung) zwischen der KV und den Krankenkassen über die Kontrastmittelpauschalen. Mit deren Abschluss dokumentieren die Krankenkassen, dass sie ihre wirtschaftlichen Interessen als gewahrt ansehen, wenn sie nicht mit höheren Ausgaben als mit denen, die sich aus der pauschalen Erstattung ergeben, belastet werden. Im Umkehrschluss bedeutet dies, dass sie auf sämtliche Vorteile (Rabatte), die aus den Marktbedingungen unterhalb des pauschalen Erstattungsbetrages resultieren, implizit verzichtet und damit keinen Anspruch haben.

Damit stellt auch die Auszahlung des Erstattungsbetrages nach der hier vertretenen Rechtsauffassung keinen Vorteil im strafrechtlichen Sinne dar. Dem Vertragsarzt bleibt auch keine andere Wahl. Durch das Wirtschaftlichkeitsgebot nach $\S 12$ Abs. 1 SGB $V$ ist er einerseits gehalten, Kontrastmittel möglichst günstig einzukaufen. Die Verpflichtung zur peinlich genauen Abrechnung, die das Bundessozialgericht in seiner ständigen Rechtsprechung annimmt (BSG, Urt. v. 24.11.1993, Az.: 6 RKa 70/91), zwingt den Vertragsarzt andererseits dazu, die in der Ergänzungsvereinbarung vorgegebenen Pseudoziffern für die Kontrastmittelverwendung einzusetzen. Es ist ihm dagegen untersagt, diese Ziffern nicht abzurechnen. Der wirtschaftliche Vorteil, der aus einer Saldierung des Einkaufspreises und des pauschalen Erstattungspreises resultiert, führt zwar zu einer Verbesserung der Lage des Empfängers, ist jedoch auf- 
grund des Anspruches auf die Erstattung des Pauschalbetrages kein strafwürdiger Vorteil.

Zudem ist festzustellen, dass die Vertragsärzte an die Bestimmungen der Gesamtverträge und damit auch der Ergänzungsvereinbarung gebunden sind, da es sich um sog. normsetzende Verträge handelt (LSG Niedersachsen-Bremen, Urt. v. 26.11.2008, Az.: L 3 KA 169/06; LSG NRW, Beschl. v. 28.12.2010, Az.: L 11 KA 60/10 B ER.). Der Radiologe ist daher nicht berechtigt, andere Beträge als die Pauschale gegenüber der KV in Rechnung zu stellen. Den Krankenkassen ist es als Körperschaft des öffentlichen Rechts nach §4 Abs. 1 SGB V zudem nicht gestattet, über die Pauschalvergütung hinaus, zusätzliche Beträge zu vereinnahmen, denn jedes Handeln der Sozialversicherungsträger, durch das Rechte und Pflichten begründet, festgestellt, geändert oder aufgehoben werden, bedarf nach §31 SGB I einer gesetzlichen Grundlage (sog. Gesetzesvorbehalt).

Gleichwohl lässt sich nicht rechtssicher bestimmen, dass die Staatsanwaltschaften oder Strafgerichte dieser restriktiven Auslegung des Vorteilsbegriffs trotz Verankerung in der $\mathrm{BGH}$-Rechtsprechung folgen werden. In Anbetracht dessen wird nachfolgend des Weiteren geprüft, ob eine Strafbarkeit aus anderen Gründen scheitern könnte.

\section{Unrechtsvereinbarung}

Das bloße Vorliegen eines „Vorteils“ genügt für die Strafwürdigkeit des Verhaltens nicht. Stattdessen muss zusätzlich eine sog. Unrechtsvereinbarung vorliegen, d. h. der „Vorteil“ muss sich unmittelbar auf eine der im Gesetz genannten Gegenleistungen des Arztes („für“) beziehen. Erforderlich ist insoweit eine Konnexitätsbeziehung.

Eine Unrechtsvereinbarung liegt vor, wenn bei der Verordnung oder der Abgabe von Arznei-, Heil- oder Hilfsmitteln oder von Medizinprodukten ( $\S 299 a$ Abs. 1 Nr. 1 StGB) oder bei der Zuführung von Patienten oder Untersuchungsmaterial (§299a Abs. 1 Nr. 3 StGB) die unlautere Bevorzugung eines anderen Wettbewerbers zumindest angestrebt wird. Es genügt mithin die Ver- ordnung (von Arznei-, Heil- oder Hilfsmitteln oder Medizinprodukten) bzw. die Zuführung (von Patienten oder Untersuchungsmaterial). Hingegen setzt §299a Abs. 1 Nr. 2 StGB den Bezug (von Arznei-, Heil- oder Hilfsmitteln oder Medizinprodukten) voraus, der jegliche Form des Sich-Verschaffens erfasst.

\section{Unlautere Bevorzugung im Wettbewerb}

Hinzutreten muss weiterhin eine „unlautere Bevorzugung im Wettbewerb“. Unter „Bevorzugung“ ist die Entscheidung zwischen zumindest zwei Bewerbern zum Nachteil eines Konkurrenten zu verstehen (Fischer, StGB, § 299 Rn. 14). Hierbei genügt es, wenn die zum Zwecke des Wettbewerbs vorgenommenen Handlungen nach der Vorstellung des Täters geeignet sind, seine eigene Bevorzugung oder die eines Dritten im Wettbewerb zu veranlassen. „Unlauter“ ist eine Bevorzugung, die geeignet ist, Mitbewerber durch Umgehung der Regeln des Wettbewerbs und durch Ausschaltung der Konkurrenz zu schädigen (Fischer, StGB, $\S 299$ Rn. 16).

Die Vertragsärzte und angestellten Ärzte unterfallen dem potenziellen Täterkreis in den Tatbestandsvarianten des Sich-Versprechen-Lassens und des Annehmens eines Vorteils bei dem Bezug von Arzneimitteln. Eine tatbestandliche Bevorzugung kommt in Betracht, wenn sich die Vertragsärzte aufgrund eines geringeren Einkaufspreises unterhalb der Kontrastmittelpauschale zur Abrechnung von Röntgenkontrastmitteln für das Angebot eines günstigeren Händlers anstatt das eines Mitbewerbers entscheiden, der einen höheren Einkaufspreis anbietet. Diese Bevorzugung im Wettbewerb ist allerdings nur dann strafwürdig, wenn sie unlauter ist, also dazu geeignet ist, Mitbewerber unter Umgehung der Regeln des Wettbewerbs zu schädigen. Das Merkmal grenzt sachwidrige von sachgemäßen Motiven für die Bevorzugung ab (Fischer, StGB, § 299 Rn. 16).

In den Ergänzungsvereinbarungen zur SSBVereinbarung wird auf die Verpflichtung zur Einhaltung des Wirtschaftlichkeitsgebotes hingewiesen. Somit kann nach hiesiger Rechtsauffassung die Sachwidrigkeit der Entscheidung für einen günstigeren Kon- trastmittellieferanten nicht bereits auf die Bevorzugung aufgrund des angebotenen Einkaufspreises desselben Kontrastmittels gestützt werden, da dieses wirtschaftliche Verhalten von Ärzten gerade erwünscht ist. Ein Vergleich der Einkaufspreise und die Entscheidung für den günstigeren ist ein sachgemäßes Motiv und damit nicht unlauter.

Zu hinterfragen ist in diesem Zusammenhang die Begründung zur Beschlussempfehlung des Ausschusses für Recht und Verbraucherschutz (Beschl. d. Ausschusses für Recht und Verbraucherschutz v. 13.4.2016, Ausschuss-Drs. 18/8106, S. 15), in der es heißt:

„Eine Strafbarkeit entfällt, wenn der Heilberufsangehörige die ihm beim Bezug gewährten Rabatte und sonstigen Vorteile zugunsten des Patienten bzw. des zuständigen Kostenträgers annimmt, um sie an diesen weiterzugeben."

Diese Ausführungen beziehen sich jedoch auf Regelungen im SGB V, die eine an sich unzulässige Zusammenarbeit zwischen Leistungserbringern und Vertragsärzten dann als zulässig ansehen, wenn sie zwischen Krankenkassen und Leistungserbringern zur Erschließung von Wirtschaftlichkeitsreserven geschlossen werden. Eine solche Regelung ist z. B. in $\S 128$ Abs. 6 S. 2 SGB V enthalten:

„Hiervon unberührt bleiben gesetzlich zulässige Vereinbarungen von Krankenkassen mit Leistungserbringern über finanzielle Anreize für die Mitwirkung an der Erschließung von Wirtschaftlichkeitsreserven und die Verbesserung der Qualität der Versorgung bei der Verordnung von Leistungen nach den $\S \S 31$ und 116b Absatz 7.“

Diese Regelung wurde durch die 15. AMGNovelle in das SGB $V$ aufgenommen. In dem Gesetzentwurf (BT-Drs. 16/12 256, S.65) wird die Regelung wie folgt begründet:

"[...] Dagegen bleibt es weiterhin zulässig, dass Krankenkassen im gesetzlich vorgesehenen Rahmen mit Leistungserbringern vereinbaren, finanzielle Anreize zu gewähren, insbesondere für eine Mitwirkung an der 
Erschließung von Wirtschaftlichkeitsreserven und einer Verbesserung der Qualität der Versorgung."

Die Regelung bestätigt die bereits getroffene Feststellung, dass Krankenkassen mit einzelnen Vertragsärzten oder auch im Rahmen der Gesamtverträge mit den KVen Vereinbarungen abschließen können, die im Ergebnis auch zu Vorteilen auf Seiten der Vertragsärzte führen. In diesem Fall fehlt es bereits an einem rechtswidrig erlangten Vorteil auf Seiten des Vertragsarztes, soweit hierfür eine gesetzliche Regelung vorhanden ist.

Eine solche Privilegierung stellt die untergesetzliche Ermächtigung an die Partner der Gesamtverträge zur Schaffung von Pauschalen in $\S 44$ Abs. 6 S. 9 BMV-Ä bzw. $\S \S 82$ Abs. 2 S. 1,83 S. 1 SGB $\vee$ dar. Durch diese Regelung wird den Gesamtvertragspartnern die Möglichkeit eingeräumt, als Ausnahme zu §44 Abs. 6 S. 7 BMV-Ä auf die Weitergabe von Vorteilen durch den Vertragsarzt zu verzichten, um langfristig auf die Preisgestaltung im Kontrastmittelmarkt Einfluss zu nehmen und damit günstigere Abgabepreise zu erzielen.

Ferner ist anhand der Gesetzesbegründung und der Beschlussbegründung des Ausschusses für Recht und Verbraucherschutz zu erkennen, dass der Gesetzgeber ganz andere Beispiele bei der Schaffung des neuen Korruptionstatbestandes vor Augen hatte. Als Beispiele für eine unmittelbare Anwendung von erworbenen Arzneimitteln werden dort die ambulante Krebstherapie und die Substitutionstherapie hervorgehoben. Da sich die oben zitierte Aussage offensichtlich auf Arzneimittel im Allgemeinen bezieht und keine Ausführungen zu einer pauschalen Kostenerstattung gemacht werden, ist davon auszugehen, dass der Gesetzgeber bei seiner Aussage zu rabattierten Arzneimitteln die Kontrastmittel nicht im Blick hatte und die Problematik im Zusammenhang mit unter dem Pauschalpreis liegenden Einkaufpreisen für Kontrastmittel nicht erkannt hat. Dies bedeutet wiederum, dass der Gesetzgeber die zitierte Aussage nicht auf Kontrastmittel bezogen hat und sie daher für die strafrechtliche Beurteilung nicht zu berücksichtigen ist.

\section{Einschränkung aufgrund des Simultanitätsprinzips}

Im Strafrecht gilt das sog. Simultanitätsprinzip gem. $\S \S 8,16$ StGB. Danach müssen Vorsatz und Tathandlung gleichzeitig vorliegen. Kauft ein Arzt aber zunächst auf Vorrat Kontrastmittel ein, weiß er zu diesem Zeitpunkt nicht, für welche konkreten Patienten er diese später verwenden wird. Insbesondere kann er nicht vorhersagen, ob dieses Kontrastmittel überhaupt für einen GKV-Patienten eingesetzt werden wird oder aber für einen PKV-Patienten. Da im letzteren Fall eine genaue Abrechnung nach Verbrauch stattfindet, kann in diesem Fall kein Vorsatz bestehen, sich einen Vorteil zu verschaffen. Folglich kann ein Vorsatz daher nicht unterstellt werden, solange dem Arzt nicht klar ist, ob er das eingekaufte Kontrastmittel für einen GKVoder PKV-Patienten einsetzen wird. Allein bei Ärzten, die ausschließlich GKV-Patienten behandeln, besteht daher die nachfolgende Problematik des vermuteten Vorsatzes. Dem Arzt müsste daher nachgewiesen werden, dass er bereits beim Einkauf der Kontrastmittel in Erwägung zog, diese später ohne Rücksicht auf die Indikation diese bei Patienten anzuwenden, bei denen entweder überhaupt keine Indikation für eine Kontrastmittelgabe oder aber für die Verwendung eines anderen Kontrastmittels vorlag. Um dem vorzubeugen, dass ein Richter jedoch zulässigerweise bei Ärzten, die ausschließlich GKV-Patienten behandeln, von den äußeren Umständen auf einen entsprechenden (bedingten) Vorsatz schließt, ist jedem Vertragsarzt - nicht nur aus ethischen und Haftungsgründen - dringend anzuraten, keine Kontrastmittel entgegen der Indikation zu verabreichen, da sich nach Einführung des §299a StGB auch eine Strafbarkeit wegen Bestechlichkeit aufdrängen würde, zusätzlich zu einer in diesem Fall ohnehin gegebenen Strafbarkeit wegen Körperverletzung.

\section{Keine Strafbarkeit nach} $\S 299$ StGB (Bestechlichkeit und Bestechung im geschäftlichen Verkehr)

Unter Bezugnahme auf die Rechtsprechung des BGH sind niedergelassene Vertragsärz- te weder als Beauftragte eines geschäftlichen Betriebs im Sinne der §299 Abs. 1, Abs. 2 StGB, noch als Amtsträger im Sinne der $\S \S 331 \mathrm{ff}$. StGB zu qualifizieren. Eine Strafbarkeit der Vertragsärzte scheidet somit grds. aus. Bei angestellten Ärzten ist diese dagegen unter bestimmten Voraussetzungen unverändert gegeben; insoweit greifen aber die vorstehenden Ausführungen zu den Tatbestandsmerkmalen des $\S 299 a$ StGB.

\section{Keine Strafbarkeit nach $\S 263$ StGB (Betrug)}

Ausgangspunkt für die Beurteilung der Frage, ob eine rechtliche Verpflichtung zur Weiterreichung von erlangten Vorteilen an die KV und/oder die Krankenkasse besteht, sind die jeweils zugrundeliegende Abrechnungsbestimmungen. Besteht, wie in $\S 44$ Abs. 6 S. 7 BMV-Ä geregelt, eine Verpflichtung des Vertragsarztes gegenüber der $\mathrm{KV}$, „die tatsächlich realisierten Preise in Rechnung zu stellen“, besteht für die rechnungsbegleichende Stelle auch die Notwendigkeit, die Abrechnung des Vertragsarztes zu prüfen. Bei einer Abrechnung nach den tatsächlichen Kosten ist der Vertragsarzt verpflichtet, ggf. vom Hersteller bzw. Lieferanten gewährte Rückvergütungen, wie Preisnachlässe, Rabatte, Umsatzbeteiligungen, Bonifikationen und rückvergütungsgleiche Gewinnbeteiligungen mit Ausnahme von Barzahlungsrabatten bis zu $3 \%$ weiterzugeben. Vertragsärzte, die entgegen diesen Vorgaben Preisnachlässe nicht weitergeben, machen sich wegen Betruges nach §263 StGB zum Nachteil der KV und ggf. der Untreue zum Nachteil der Krankenkassen nach § 266 StGB strafbar:

„Wer in dieser Weise zur Angabe seiner individuell errechneten tatsächlichen Kosten verpflichtet ist, gleichwohl aber ohne jede Berechnung und ohne jede eigene substantiierte Kalkulation pauschal ,Beträge geltend macht (e), die nach seiner Auffassung die tatsächlich entstandenen Kosten ausmachten [...], der täuscht. “ (BGH, Urt.v. 15.10.1991, Az.: 4 StR 420/91).

Der Vertragsarzt hat außerdem die Richtigkeit seiner Abrechnung durch Unterschrift gegenüber der rechnungsbegleichenden 
Stelle zu bestätigen (vgl. §44 Abs. 6 S. 8 BMV-Ä). Als Ausnahme von diesem Grundsatz wird die Vereinbarung von Pauschalbeträgen im Sachkostenbereich durch die Vertragspartner der Gesamtverträge angesehen. In diesen Fällen ist der gesamtvertraglich vereinbarte Betrag abrechnungsfähig, unabhängig davon, welche Kosten dem Vertragsarzt tatsächlich entstanden sind (vgl. Trieb, in: Schiller, Bundesmantelvertrag Ärzte, 2014, §44 Rn. 19).

Erfolgt die Abrechnung der Kontrastmittel auf der Grundlage von Pauschalvergütungen, ist der Betrugstatbestand nicht erfüllt, wenn der Vertragsarzt seinen Einkaufsvorteil nicht an die Krankenkasse weiterreicht. Es fehlt insoweit an einer tatbestandlichen Täuschung über eine Tatsache. Rechnet ein Arzt daher wie in der Kontrastmittelvereinbarung über eine Pseudoziffer eine Kontrastmittelpauschale ab, täuscht er sein Gegenüber, in diesem Fall die KV, nicht darüber, dass er das Kontrastmittel zu vergünstigten Konditionen eingekauft hat. Durch die Einführung einer Pauschale nimmt die KV gerade in Kauf, dass der Arzt tatsächlich weniger oder aber auch mehr als den Pauschalbetrag für den Kontrastmitteleinkauf ausgegeben hat. Sie möchte durch eine solche Pauschale gerade nicht über den jeweiligen exakten Einkaufspreis informiert werden und überlässt es ihren Vertragsärzten, Preise mit Kontrastmittelhändlern zu verhandeln. Es fehlt damit an einem unwahren Erklärungsinhalt, wenn der Arzt die Pauschale abrechnet. Er erklärt damit nämlich allein, dass er Kontrastmittel verwendet hat, aber nicht wie viel und zu welchem Preis er dieses eingekauft hat.
Eine Strafbarkeit aus $\S 263$ StGB kommt nur dann in Betracht, wenn der Vertragsarzt Kontrastmittel abrechnet, ohne sie tatsächlich beim Patienten eingesetzt zu haben, nicht aber für das Nichtweiterreichen eines Einkaufsvorteils. Dies folgt schon aus der Pflicht zur peinlich genauen Abrechnung. Danach ist es einem Vertragsarzt verwehrt, die Pauschale nicht abzurechnen, wenn er Kontrastmittel tatsächlich verwendet hat.

\section{Fazit}

Die größte Herausforderung in der Praxis wird zukünftig die Grenzziehung zwischen einem straffreiem und einem strafrechtlich missbilligtem Verhalten nach § 299a StGB sein, da sich die strafrechtlichen Grenzen gegenwärtig nicht rechtsverbindlich bestimmen lassen. Rechtssicherheit wird vielmehr sukzessiv im Laufe der Zeit durch die Rechtsprechung und Handhabungspraxis der Strafverfolgungsbehörden entstehen.

Nach der hier vertretenen Rechtsauffassung ist eine Verurteilung wegen Bestechlichkeit im Gesundheitswesen durch Vertragsärzte, die Kontrastmittel unterhalb der festgelegten Pauschalbeträge einkaufen und den Einkaufsvorteil nicht an die Krankenkassen weiterreichen, nicht zu befürchten, da es neben dem Tatbestandsmerkmal des „Vorteils“ regelmäßig an der Unlauterkeit fehlen wird, solange der Arzt seine heilberuflichen Entscheidungen sorgfältig trifft und für jeden Patienten individuell prüft, ob und welches Kontrastmittel einzusetzen ist und nicht aus wirtschaftlichen Motiven einem Patienten Kontrast- mittel verabreicht, obwohl es für die beabsichtigte Bilddiagnostik nicht indiziert gewesen ist.

Diese Ansicht findet auch Schrifttum ihre Bestätigung. So führen Brettel/Mand (A\&R 2016, 99) aus:

„Bei einem solchen Pauschbetrag besteht nach §44 Abs. 6 Satz 9 BMV-Ärzte keine Weitergabepflicht etwaiger Einkaufsvorteile mehr. Das heißt, der Arzt ist frei, zwischen Anbietern auszuwählen. Wenn er dabei Rabatte oder andere Vorteile angeboten bekommt und annimmt, so mag dies seine Auswahlentscheidung anleiten und womöglich eine Unterversorgung durch eine Bevorzugung von „Billiganbietern“ begünstigen. Korruption ist dies jedoch nicht, jedenfalls wenn man die Perspektive auf das Verhältnis zwischen Kassen und Kassenärzten konzentriert. "

Abschließend ist zu bemerken, dass in der Zwischenzeit mehrere Kassenärztliche Vereinigungen Stellungnahmen vorgelegt haben, in denen sie ebenfalls zu dem Ergebnis kommen, dass der Einkauf von Röntgenkontrastmitteln zu Preisen unter dem pauschalen Erstattungsniveau (weiterhin) nicht strafbar ist.

Prof. Dr. Peter Wigge
Rechtsanwalt
Fachanwalt für Medizinrecht
Rechtsanwälte Wigge
Scharnhorststraße 40
48151 Münster
++49/251/535950
++49/251/5359599
kanzlei@ra-wigge.de

